\title{
Health Status
}

National Cancer Institute

\section{Source}

National Cancer Institute. Health Status. NCI Thesaurus. Code C16669.

The state of a subject's mental or physical condition. 